06

\title{
Генерация второй гармоники-суммарной частоты в тонком сферическом слое. II. Анализ диаграмм направленности
}

\author{
(C) А.И. Толкачёв, В.Н. Капшай, А.А. Шамына
}

Гомельский государственный университет им. Ф. Скорины, 246019 Гомель, Республика Беларусь

e-mail: anton.talkachov@gmail.com, kapshai@rambler.ru, anton.shamyna@gmail.com

Поступила в редакцию 06.06.2021 г.

В окончательной редакции 23.08.2021 г.

Принята к публикации 02.09.2021 г.

\begin{abstract}
Проведен графический анализ решения задачи о генерации второй гармоники-суммарной частоты в тонком сферическом слое при его облучении двумя когерентными плоскими электромагнитными волнами с эллиптическими поляризациями и одинаковыми частотами. Определены асимметрии, количественно описывающие форму трехмерных диаграмм направленности (пространственное распределение плотности мощности излучения второй гармоники-суммарной частоты). Трехмерные диаграммы направленности и асимметрии проанализированы для различных комбинаций параметров: отношения комплексных амплитуд падающих волн, угла между волновыми векторами падающих волн (угла раскрытия), эллиптичности, ориентации эллипсов поляризации, размеров сферической частицы. Обнаружено, что при малых размерах частицы каждому типу анизотропии соответствует своя индивидуальная форма диаграммы направленности. Установлено, что для одного из типов анизотропии форма диаграммы направленности практически не изменяется при варьировании указанных выше параметров почти во всех возможных диапазонах.
\end{abstract}

Ключевые слова: генерация второй гармоники, генерация суммарной частоты, диэлектрическая сферическая частица, асимметрия диаграммы направленности, тензор нелинейной восприимчивости.

DOI: $10.21883 / \mathrm{OS} .2021 .12 .51743 .2386-21$

\section{Явный вид векторов в задаче}

Ось $O z$ декартовой системы координат удобно направить вдоль суммы векторов $\mathbf{k}^{(1)}$ и $\mathbf{k}^{(2)}$, которые, не нарушая общности, будем считать находящимися в плоскости $O y z$ (рис. 1,a). Угол между волновыми векторами $\mathbf{k}^{(1)}$ и $\mathbf{k}^{(2)}$, который также называют углом раскрытия [1], обозначим $\gamma$. Так как длины волновых векторов равны, то они будут расположены под одинаковыми углами $\gamma_{1}$ и $\gamma_{2}$ к оси $O z$, за исключением случая, когда $\left|\mathbf{k}^{(1)}+\mathbf{k}^{(2)}\right|=0$. Таким образом,

$$
\begin{aligned}
& \gamma_{1}=\gamma_{2}=\gamma / 2 \quad \text { при } \quad \gamma \in[0, \pi), \\
& \gamma_{1}=0, \gamma_{2}=\pi \quad \text { при } \quad \gamma=\pi .
\end{aligned}
$$

Такое определение угла раскрытия сделано для соответствия предельному переходу от генерации суммарной частоты [2] к генерации второй гармоники-суммарной частоты.

Волновые векторы источников и соответствующие комплексные векторы поляризации во введенной системе координат по аналогии с формулами в работе [2] имеют вид

$$
\mathbf{k}^{(\alpha)}=k_{\omega}\left((-1)^{\alpha} \sin \gamma_{\alpha} \mathbf{e}_{y}+\cos \gamma_{\alpha} \mathbf{e}_{z}\right),
$$

$$
\begin{aligned}
\mathbf{e}^{(\alpha)} & =\left[\left(\cos \varphi_{\mathrm{in}}^{(\alpha)}-i \sigma_{\alpha} \sin \varphi_{\mathrm{in}}^{(\alpha)}\right) \mathbf{e}_{x}\right. \\
& +\cos \gamma_{\alpha}\left(i \sigma_{\alpha} \cos \varphi_{\mathrm{in}}^{(\alpha)}+\sin \varphi_{\mathrm{in}}^{(\alpha)}\right) \mathbf{e}_{y}+(-1)^{1+\alpha} \sin \gamma_{\alpha} \\
& \left.\times\left(i \sigma_{\alpha} \cos \varphi_{\mathrm{in}}^{(\alpha)}+\sin \varphi_{\mathrm{in}}^{(\alpha)}\right) \mathbf{e}_{z}\right]\left(1+\sigma_{\alpha}^{2}\right)^{-1 / 2},
\end{aligned}
$$

где $\mathbf{e}_{x}, \mathbf{e}_{y}, \mathbf{e}_{z}-$ базисные векторы декартовой системы координат, $\sigma_{\alpha}-$ величины, характеризующие эллиптичности падающих волн $\left(\sigma_{\alpha}>0-\right.$ правая поляризация, $\sigma_{\alpha}<0-$ левая поляризация, $\sigma_{\alpha}=0-$ линейная поляризация), $\varphi_{\text {in }}^{(\alpha)}$ - углы между осью $O x$ и большими полуосями эллипсов поляризации соответствующих падающих электромагнитных волн. Базисный вектор $\mathbf{e}_{x}$, действительная часть вектора поляризации $\mathbf{e}^{(\alpha)}$ и соответствующий волновой вектор $\mathbf{k}^{(\alpha)}$ должны образовывать правую тройку векторов при $0<\varphi<\pi$ и левую при $-\pi<\varphi<0$.

В рамках обсуждаемой модели можно учитывать дисперсию, для чего понадобится коэффициент

$$
\xi=\frac{k_{2 \omega}}{2 k_{\omega}}=\frac{n_{2 \omega}}{n_{\omega}} .
$$

С учетом этого векторы рассеяния в сферической системе координат и их модули имеют вид

$$
\begin{aligned}
& \mathbf{q}^{(11)}=2 \mathbf{k}^{(1)}-\mathbf{k}^{(2 \omega)}=-2 k_{\omega}\left[\left(\sin \gamma_{1} \sin \theta \sin \varphi\right.\right. \\
& \left.-\cos \gamma_{1} \cos \theta+\xi\right) \mathbf{e}_{r}+\left(\sin \gamma_{1} \cos \theta \sin \varphi+\cos \gamma_{1} \sin \theta\right) \mathbf{e}_{\theta} \\
& \left.+\sin \gamma_{1} \cos \varphi \mathbf{e}_{\varphi}\right]
\end{aligned}
$$



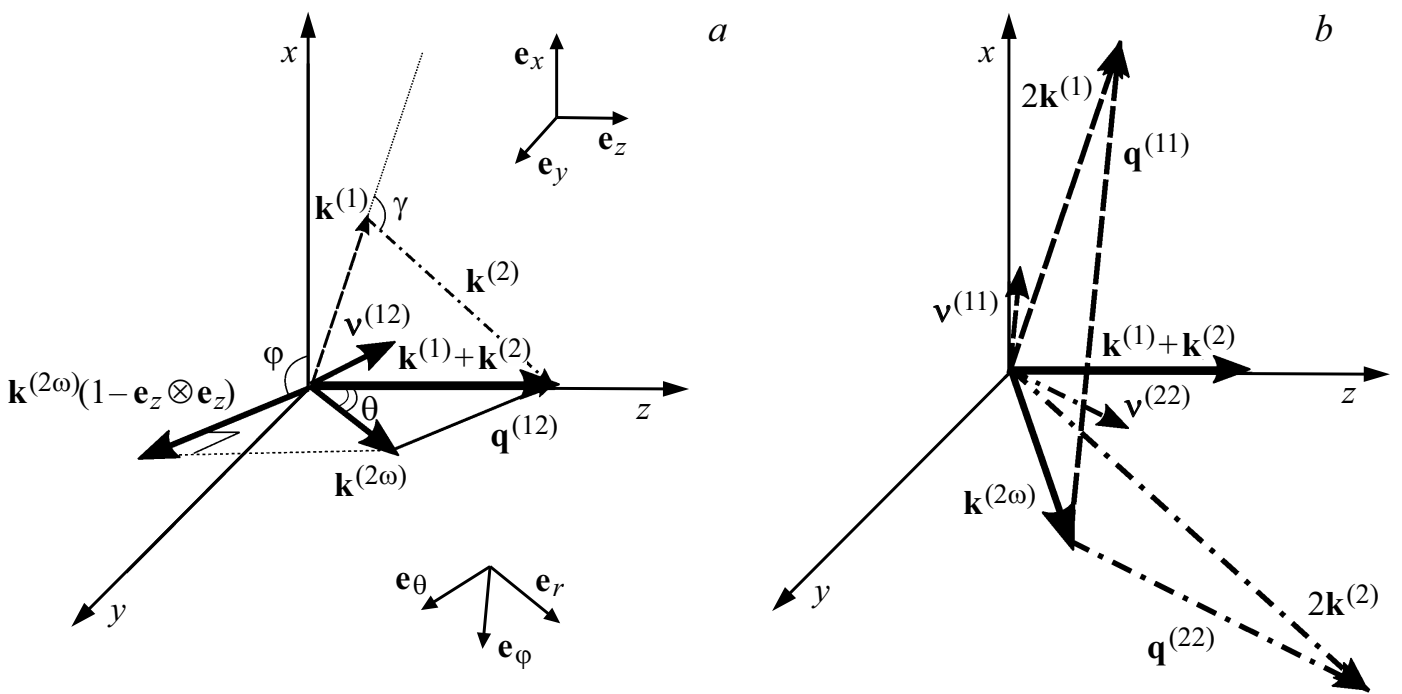

Рис. 1. Схема векторов, используемых в задаче: $(a)$ векторы, необходимые для получения $\mathbf{q}^{(12)},(b)$ векторы, необходимые для получения $\mathbf{q}^{(11)}$ и $\mathbf{q}^{(22)}$. Штриховыми линиями обозначены векторы, относящиеся к первой волне, штрихпунктирными линиями относящиеся ко второй волне.

$$
\begin{gathered}
\mathbf{q}^{(12)}=\mathbf{q}^{(21)}=\mathbf{k}^{(1)}+\mathbf{k}^{(2)}-\mathbf{k}^{(2 \omega)} \\
=-2 k_{\omega}\left[(\xi-\xi \cos \theta) \mathbf{e}_{r}+\xi \sin \theta \mathbf{e}_{\theta}\right], \\
\mathbf{q}^{(22)}=2 \mathbf{k}^{(2)}-\mathbf{k}^{(2 \omega)}=2 k_{\omega}\left[\left(\sin \gamma_{2} \sin \theta \sin \varphi\right.\right. \\
\left.+\cos \gamma_{2} \cos \theta-\xi\right) \mathbf{e}_{r}+\left(\sin \gamma_{2} \cos \theta \sin \varphi-\cos \gamma_{2} \sin \theta\right) \mathbf{e}_{\theta} \\
\left.+\sin \gamma_{2} \cos \varphi \mathbf{e}_{\varphi}\right],
\end{gathered}
$$

$q^{(11)}=2 k_{\omega} \sqrt{1+\xi^{2}-2 \xi \cos \gamma_{1} \cos \theta+2 \xi \sin \gamma_{1} \sin \theta \sin \varphi}$,

$q^{(12)}=q^{(21)}=2 k_{\omega} \sqrt{\xi^{2}+\xi^{2}-2 \zeta \xi \cos \theta}$,

$q^{(22)}=2 k_{\omega} \sqrt{1+\xi^{2}-2 \xi \cos \gamma_{2} \cos \theta-2 \xi \sin \gamma_{2} \sin \theta \sin \varphi}$.

Аналогично работе [2] определим величину $\xi$ следующим образом:

$$
\left|\mathbf{k}^{(1)}+\mathbf{k}^{(2)}\right|=2 k_{\omega} \xi
$$

В рассматриваемом здесь случае, очевидно, $\xi=\cos (\gamma / 2)$. Тогда единичные векторы $\boldsymbol{v}^{(\alpha \beta)}$ в направлении векторов $\mathbf{q}^{(\alpha \beta)}$ запишутся с учетом (4) и (5) в виде

$$
\begin{gathered}
\boldsymbol{v}^{(11)}=\frac{\mathbf{q}^{(11)}}{q^{(11)}}=\left(\left(\cos \gamma_{1} \cos \theta-\sin \gamma_{1} \sin \theta \sin \varphi-\xi\right) \mathbf{e}_{r}\right. \\
\left.-\left(\sin \gamma_{1} \cos \theta \sin \varphi+\cos \gamma_{1} \sin \theta\right) \mathbf{e}_{\theta}-\sin \gamma_{1} \cos \varphi \mathbf{e}_{\varphi}\right) \\
\times\left(1+\xi^{2}-2 \xi \cos \gamma_{1} \cos \theta+2 \xi \sin \gamma_{1} \sin \theta \sin \varphi\right)^{-1 / 2}, \\
\boldsymbol{v}^{(12)}=\boldsymbol{v}^{(21)}=\frac{\mathbf{q}^{(12)}}{q^{(12)}}=\frac{(\xi \cos \theta-\xi) \mathbf{e}_{r}-\xi \sin \theta \mathbf{e}_{\theta}}{\sqrt{\xi^{2}+\xi^{2}-2 \xi \xi \cos \theta}},
\end{gathered}
$$

$$
\begin{gathered}
\boldsymbol{v}^{(22)}=\frac{\mathbf{q}^{(22)}}{q^{(22)}}=\left(\left(\sin \gamma_{2} \sin \theta \sin \varphi+\cos \gamma_{2} \cos \theta-\xi\right) \mathbf{e}_{r}\right. \\
\left.+\left(\sin \gamma_{2} \cos \theta \sin \varphi-\cos \gamma_{2} \sin \theta\right) \mathbf{e}_{\theta}+\sin \gamma_{2} \cos \varphi \mathbf{e}_{\varphi}\right) \\
\times\left(1+\xi^{2}-2 \xi \cos \gamma_{2} \cos \theta-2 \xi \sin \gamma_{2} \sin \theta \sin \varphi\right)^{-1 / 2}
\end{gathered}
$$

\section{Влияние параметров задачи на пространственное распределение генерируемого излучения}

\section{Анализируемые параметры}

Проведем анализ формы диаграмм направленности генерируемого излучения и связанных с ними характеристик в зависимости от значений различных варьируемых параметров для отдельных типов тензора нелинейной диэлектрической восприимчивости.

При анализе свойств генерируемого излучения удвоенной частоты в теоретических работах и при обработке экспериментальных данных исследователи часто рассматривают некоторые частные случаи тензора нелинейной диэлектрической восприимчивости. Так, в paботах $[3,4]$ в тензоре нелинейной диэлектрической восприимчивости отличным от нуля полагался только коэффициент $\chi_{\perp \perp \perp}^{(2)}$ (в наших обозначениях $\chi_{1}^{(2)} \neq 0$, $\left.\chi_{2-4}^{(2)}=0\right)$. Авторы работы [5] при изучении явления генерации второй гармоники от поверхности сферической частицы для построения зависимостей интенсивности второй гармоники от полярного угла наблюдения выбрали частный случай тензора $\chi_{\perp \perp \perp}^{(2)} \neq 0 \quad\left(\chi_{1}^{(2)} \neq 0\right.$, $\left.\chi_{2-4}^{(2)}=0\right)$. В работе [6] рассматривали случай не равного нулю коэффициента $\chi_{\perp\|\|}^{(2)}\left(\chi_{1}^{(2)} / \chi_{2}^{(2)}=-1, \chi_{3,4}^{(2)}=0\right)$, a также комбинации коэффициентов $\chi_{\perp \perp \perp}^{(2)}, \chi_{\perp\|\|}^{(2)}, \chi_{\|\perp\|}^{(2)}$, 
соответствующих малахитовому зеленому. Авторы работы [7] для теоретического исследования углового распределения интенсивности использовали три частных случая тензора нелинейной диэлектрической восприимчивости: $\chi_{\perp \perp \perp}^{(2)}\left(\chi_{1}^{(2)} \neq 0, \chi_{2-4}^{(2)}=0\right), \chi_{\perp\|\| \|}^{(2)}\left(\chi_{1}^{(2)} / \chi_{2}^{(2)}=-1\right.$, $\left.\chi_{3,4}^{(2)}=0\right)$ и $\chi_{\|\perp\|}^{(2)}\left(\chi_{1}^{(2)} / \chi_{3}^{(2)}=-2, \chi_{2,4}^{(2)}=0\right)$. В этой работе была отмечена близость теоретических результатов при использовании нелинейной модели Рэлея-Ганса-Дебая с отличным от нуля коэффициентом $\chi_{\perp\|\|}^{(2)}$ и экспериментальных результатов, полученных для частиц радиуса $100 \mathrm{~nm}$ и менее, покрытых малахитовым зеленым.

Следуя данным методикам исследования нелинейных свойств поверхностных слоев, мы проанализируем влияние отдельных типов анизотропии на генерируемое излучение. Здесь и в дальнейшем под типом анизотропии $\chi_{i}^{(2)}$ подразумеваются значения независимых компонент тензора нелинейной диэлектрической восприимчивости второго порядка $\chi_{i}^{(2)}=1,\left.\chi_{j}^{(2)}\right|_{j \neq i}=0$. Любая комбинация этих компонет является отдельным типом анизотропии со своими уникальными свойствами, но мы рассмотрим только наиболее простые типы тензора $\chi_{i j k}^{(2)}$ : типы анизотропии $\chi_{1}^{(2)}, \chi_{2}^{(2)}, \chi_{3}^{(2)}, \chi_{4}^{(2)}$ и тензор $\chi_{i j k}^{(2)}$, соответствующий малахитовому зеленому.

Анализ генерируемого излучения при всех возможных значениях параметров задачи является крайне трудоемкой задачей, поэтому рассмотрим случай генерации в поверхностном слое малой частицы. По умолчанию характеристики частицы и параметры падающих волн зададим в виде

$$
\begin{gathered}
k_{\omega} a=0.1, \quad \xi=1.34 / 1.33, \quad \gamma=0.5, \\
\sigma_{1}=\sigma_{2}=0, \quad \eta=1, \quad \varphi_{\text {in }}^{(1)}=\pi / 2, \quad \varphi_{\text {in }}^{(2)}=0 .
\end{gathered}
$$

Здесь $k_{\omega} a-$ накопленная разность фаз при прохождении падающей волной расстояния, равного радиусу частицы, угол между волновыми векторами $\mathbf{k}^{(1)}$ и $\mathbf{k}^{(2)}$ равен 0.5 , исходные волны линейно поляризованы, их комплексные амплитуды напряженности одинаковы, направления векторов поляризации составляют углы $\pi / 2$ и 0 с осью $O x$ соответственно. Показатель дисперсии соответствует отношению показателей преломления для воды на длинах волн 400 и $800 \mathrm{~nm}$ в вакууме [8]. Излучение этих длин волн было использовано, например, в работе [6], посвященной экспериментальному исследованию генерации второй гармоники. При этом фиксированными параметрами задачи в данной части работы являются $k_{\omega}, \sigma_{1}, \xi$.

Для удобства количественного сравнения диаграмм направленности введем вспомогательные показатели (по аналогии с [9]) и назовем их асимметриями (вперед-назад (FB), вправо-влево (RL) и вверх-вниз (UD)):

$$
A_{\mathrm{FB}}=\frac{\int_{\Omega_{\mathrm{F}}} S_{r}^{(2 \omega)} d \Omega-\int_{\Omega_{\mathrm{B}}} S_{r}^{(2 \omega)} d \Omega}{\int_{4 \pi} S_{r}^{(2 \omega)} d \Omega},
$$

$$
\begin{aligned}
& A_{\mathrm{RL}}= \frac{\int_{\Omega_{\mathrm{R}}} S_{r}^{(2 \omega)} d \Omega-\int_{\Omega_{\mathrm{L}}} S_{r}^{(2 \omega)} d \Omega}{\int_{4 \pi} S_{r}^{(2 \omega)} d \Omega}, \\
& A_{\mathrm{UD}}=\frac{\int_{\Omega_{\mathrm{U}}} S_{r}^{(2 \omega)} d \Omega-\int_{\Omega_{\mathrm{D}}} S_{r}^{(2 \omega)} d \Omega}{\int_{4 \pi} S_{r}^{(2 \omega)} d \Omega} .
\end{aligned}
$$

Здесь $\Omega_{\mathrm{F}}\left(\Omega_{\mathrm{B}}\right)$ - телесный угол, ограниченный передней (задней) полусферой, $\Omega_{\mathrm{R}}\left(\Omega_{\mathrm{L}}\right)$ - телесный угол, ограниченный правой (левой) полусферой, $\Omega_{\mathrm{U}}\left(\Omega_{\mathrm{D}}\right)-$ телесный угол, ограниченный верхней (нижней) полусферой. То есть

$$
\begin{gathered}
\Omega_{\mathrm{F}}: \theta \in\left[0 ; \frac{\pi}{2}\right), \quad \Omega_{\mathrm{B}}: \theta \in\left[\frac{\pi}{2} ; \pi\right], \\
\Omega_{\mathrm{R}}: \varphi \in(0 ; \pi], \quad \Omega_{\mathrm{L}}: \varphi \in(-\pi ; 0], \\
\Omega_{\mathrm{U}}: \varphi \in\left(-\frac{\pi}{2} ; \frac{\pi}{2}\right], \quad \Omega_{\mathrm{D}}: \varphi \in\left(-\pi ;-\frac{\pi}{2}\right] \cup\left(\frac{\pi}{2} ; \pi\right] .
\end{gathered}
$$

\section{Диаграммы направленности}

Приведем теперь нормированные диаграммы направленности генерируемого излучения для различных значений независимых компонент тензора $\chi_{i j k}^{(2)}$ (в том числе для значений этих компонент, измеренных в эксперименте для малахитового зеленого [6]) при значениях параметров задачи (8) (рис. 2).

1) Для типа анизотропии $\chi_{1}^{(2)}$ (рис. 2,a) на диаграмме имеются два лепестка с преимущественным излучением в переднюю полусферу $\left(A_{\mathrm{FB}}=0.347\right)$. Возрастание $\gamma$ до 2.5 (рис. 3,a) приводит к увеличению размера нижнего лепестка и ориентации его в сторону задней полусферы, при этом $A_{\mathrm{FB}}=-0.297$.

При углах раскрытия $\gamma<1.38$ большая часть генерируемого излучения направлена в переднюю полусферу, при $\gamma>1.38-$ в заднюю (при этом минимум $A_{\mathrm{FB}}$ наблюдается при $\gamma \approx 2.38$ ), что можно видеть из рис. $4, a$. Значения для асимметрии RL (рис. 4,b) отрицательны, т.е. в левую полусферу генерируется больше энергии. График $A_{\mathrm{RL}}(\gamma)$ имеет явный минимум $\left(A_{\mathrm{RL}} \approx-0.35\right)$ при $\gamma \approx 1.40$. Аналогичный вид зависимости наблюдается и для $A_{\mathrm{UD}}$ (рис. $4, c$, минимум $A_{\mathrm{UD}} \approx-0.39$ при $\gamma \approx 2.05$ ), т. е. в нижнюю полусферу генерируется больше энергии, чем в верхнюю.

При изменении параметра $|\eta|$ (рис. 3,b), а именно удалении от значения $|\eta|=1$ в любом направлении, форма диаграммы направленности приближается к аналогичной для генерации второй гармоники при падении одной волны [10], что показано аналитически в формуле (27) части I. Изменение разности фаз $(\arg \eta)$ для данного типа анизотропии может привести к увеличению количества лепестков и значительному изменению их формы, что можно видеть из рис. 3,c. При этом 


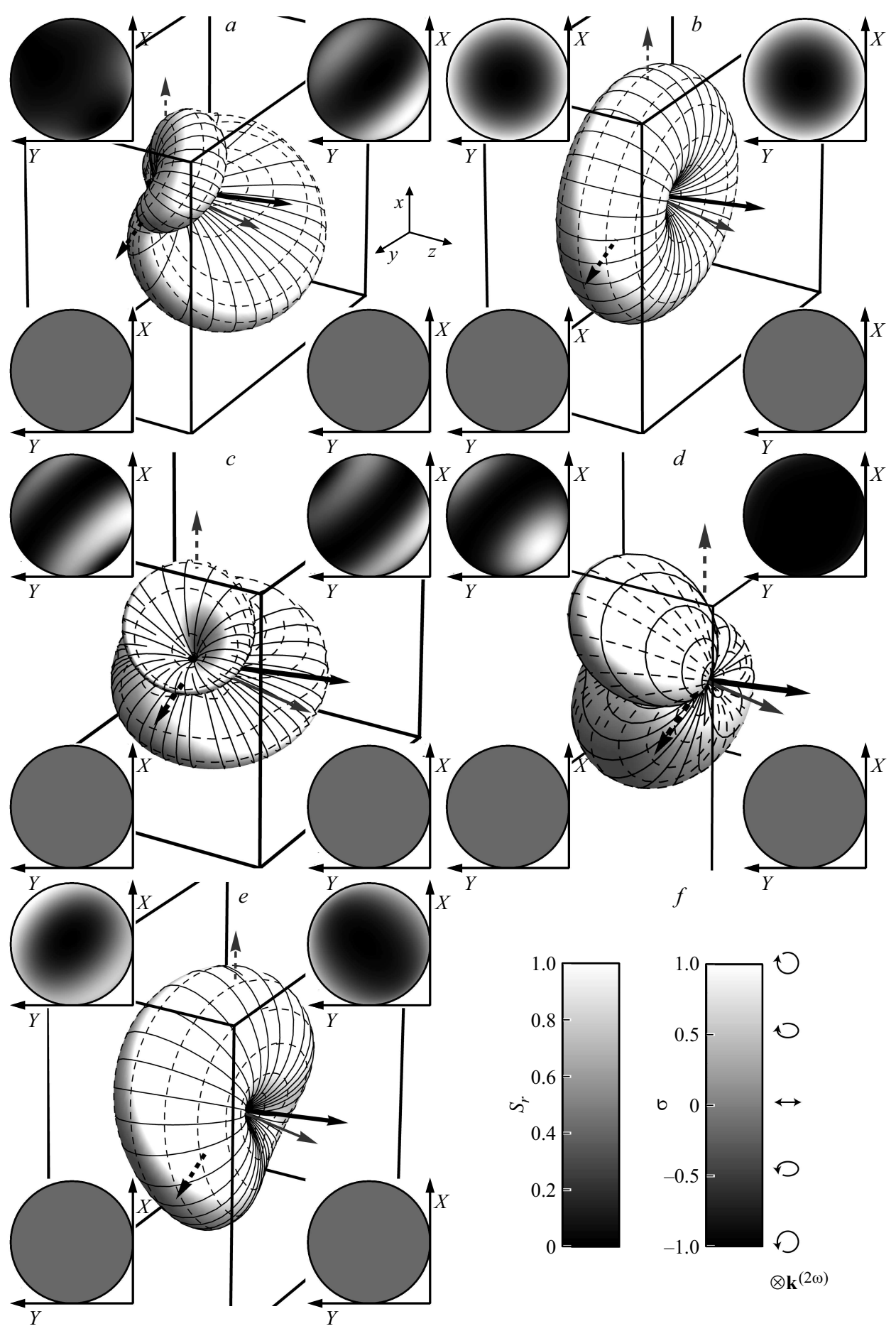

Рис. 2. Трехмерные нормированные диаграммы направленности для генерации второй гармоники-суммарной частоты от сферического слоя. $k_{\omega} a=0.1, \sigma_{1}=\sigma_{2}=0, \eta=1, \varphi_{\text {in }}^{(1)}=\pi / 2, \varphi_{\text {in }}^{(2)}=0, \gamma=0.5$. Легенда представлена на фрагменте $f$. Штриховые стрелки направлены вдоль больших полуосей эллипсов поляризации падающих волн. Утолщенные черные стрелки относятся к первой волне, тонкие серые - ко второй. Проекции больших и меньших полуосей эллипса поляризации генерируемых волн на поверхность диаграммы направленности являются касательными к сплошным и штриховым линиям на ее поверхности соответственно. В верхней части диаграммы направленности расположены ее проекции на плоскость $O x y(л е в а я: ~ \pi / 2<\theta<\pi$, правая: $0<\theta<\pi / 2$ ). Белый цвет соответствует максимальной мощности излучения в данном направлении, черный цвет соответствует отсутствию излучения в данном направлении. В нижней части диаграммы направленности расположены графики, характеризующие эллиптичность генерируемого излучения с тем же правилом проецирования. На этих графиках белый цвет соответствует правоциркулярно поляризованному излучению, черный цвет - левоциркулярно поляризованному излучению, серый цвет - линейной поляризации излучения. Типы анизотропии: $(a) \chi_{1}^{(2)},(b) \chi_{2}^{(2)},(c) \chi_{3}^{(2)},(d) \chi_{4}^{(2)},(e)$ малахитовый зеленый. 


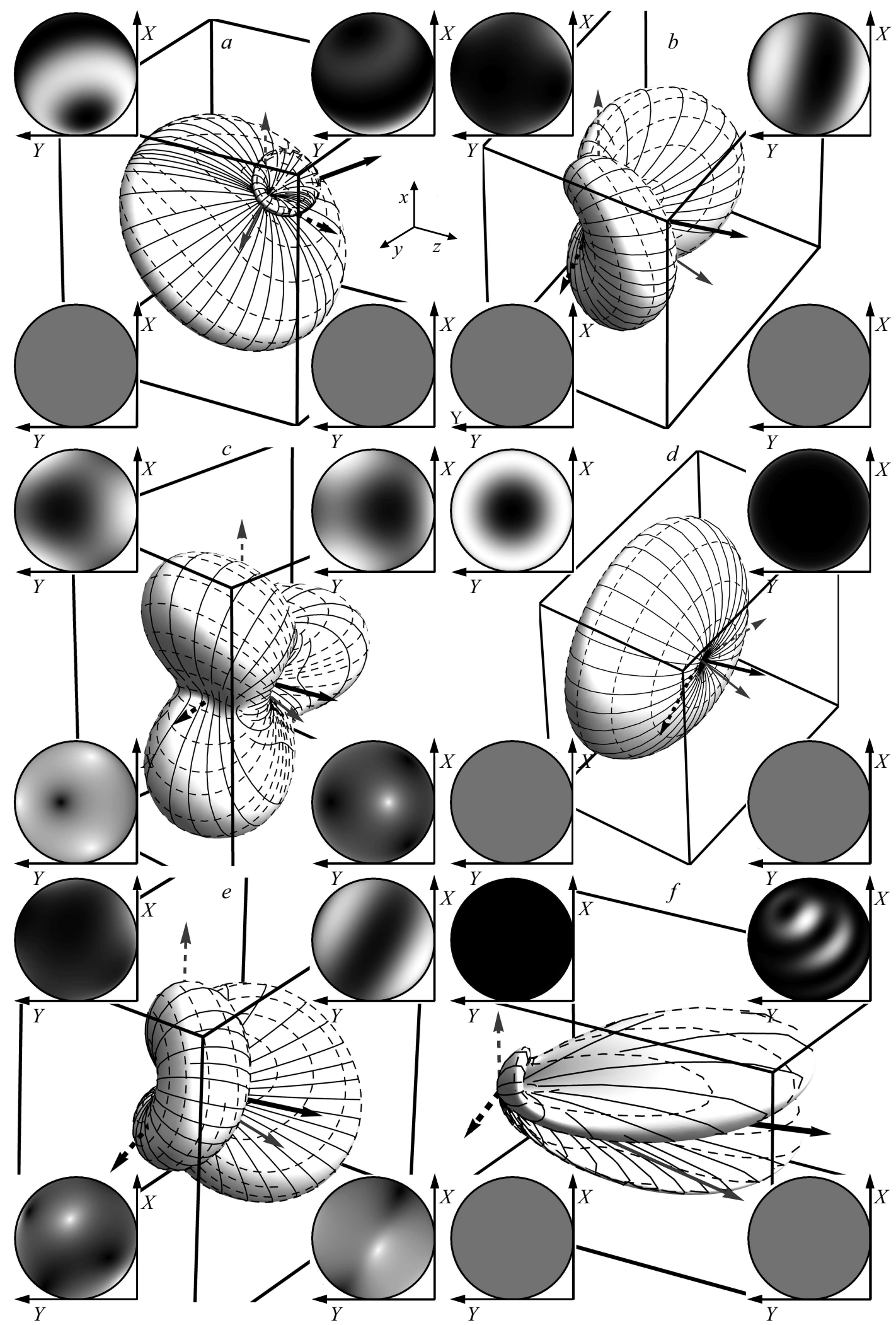

Рис. 3. Трехмерные нормированные диаграммы направленности для генерации второй гармоники-суммарной частоты от сферического слоя с типом анизотропии $\chi_{1}^{(2)}$. Обозначения такие же, как и на рис. 2. Общие для всех диаграмм направленности параметры: $\sigma_{1}=0, \varphi_{\mathrm{in}}^{(1)}=\pi / 2$. Индивидуальные параметры построения: $(a) k_{\omega} a=0.1, \sigma_{2}=0, \eta=1, \varphi_{\mathrm{in}}^{(2)}=0, \gamma=2.5,(b) k_{\omega} a=0.1, \sigma_{2}=0$, $\eta=0.25, \varphi_{\text {in }}^{(2)}=0, \gamma=0.5,(c) k_{\omega} a=0.1, \sigma_{2}=0,|\eta|=1, \arg \eta=\pi / 2, \varphi_{\text {in }}^{(2)}=0, \gamma=0.5,(d) k_{\omega} a=0.1, \sigma_{2}=0, \eta=1, \varphi_{\text {in }}^{(2)}=-\pi / 2$, $\gamma=0.5,(e) k_{\omega} a=0.1, \sigma_{2}=1, \eta=1, \varphi_{\text {in }}^{(2)}=0, \gamma=0.5,(f) k_{\omega} a=2.79, \sigma_{2}=0, \eta=1, \varphi_{\text {in }}^{(2)}=0, \gamma=0.5$. 

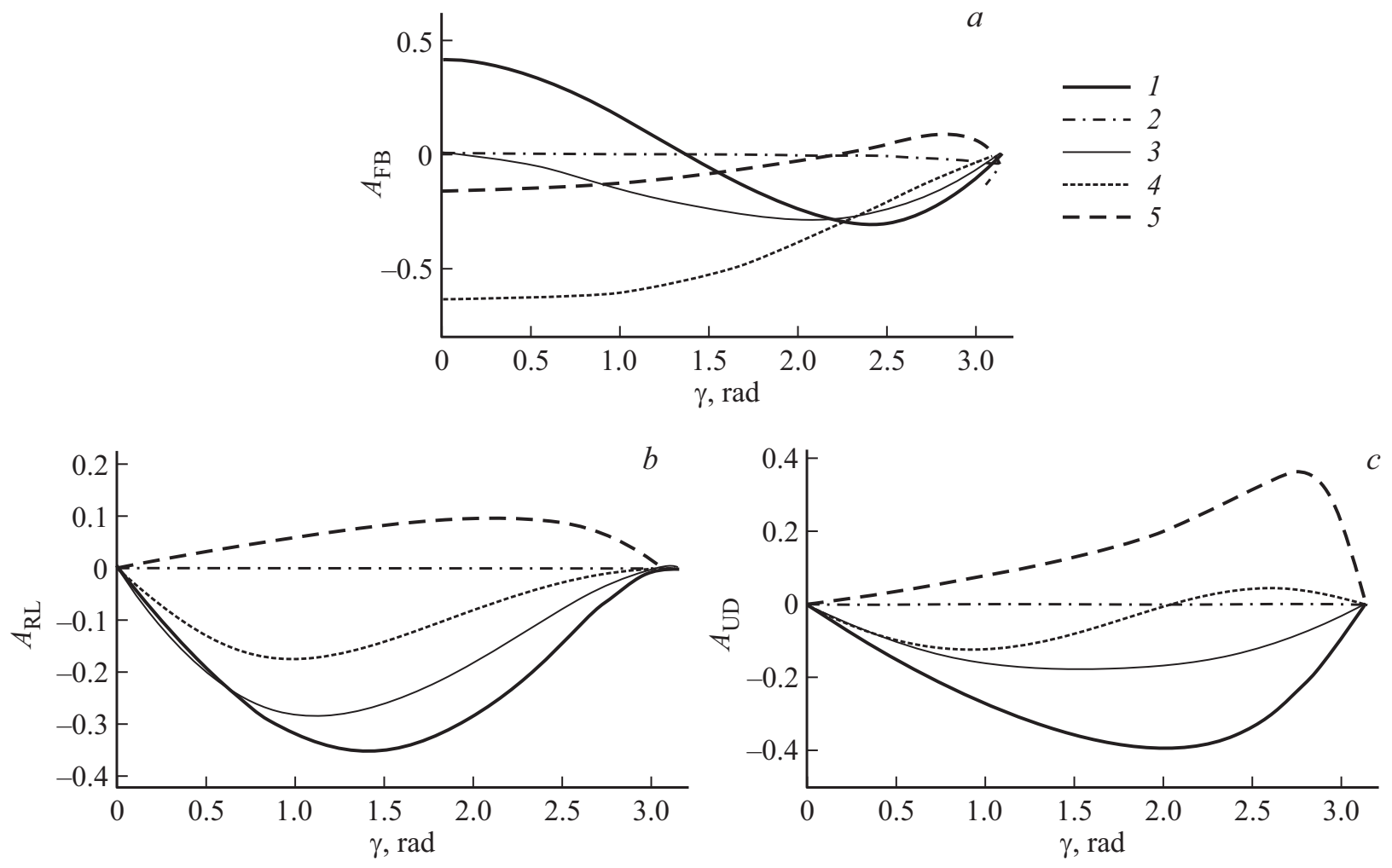

Рис. 4. Зависимость асимметрий генерируемого излучения от угла раскрытия: (a) вперед-назад, $(b)$ вправо-влево, (c) вверх-вниз. Типы анизотропии: $(1) \chi_{1}^{(2)},(2) \chi_{2}^{(2)},(3) \chi_{3}^{(2)},(4) \chi_{4}^{(2)},(5)$ малахитовый зеленый.

генерируется эллиптически поляризованное излучение с преимущественно правой поляризацией в переднюю полусферу и преимущественно левой поляризацией в заднюю. Такой же вывод об изменении формы диаграммы направленности можно сделать исходя из графиков для асимметрий (рис. 5).

Поворот вектора поляризации второй волны $\left(\varphi_{\text {in }}^{(2)}\right.$, pис. $3, d)$ в области положительных $\varphi_{\text {in }}^{(2)}\left(0 \leq \varphi_{\text {in }}^{(2)} \leq \pi\right)$ практически не вызывает изменений асимметрий FB и UD, но при отрицательных $\varphi_{\text {in }}^{(2)}\left(-\pi<\varphi_{\text {in }}^{(2)}<0\right)$ графики для всех асимметрий имеют ярко выраженные экстремумы (рис. 6), при этом диаграмма направленности имеет один главный лепесток, направленный в заднюю полусферу (рис. 3, $d$ ).

Изменение эллиптичности каждой из волн (рис. $3, e$ ) приводит к уменьшению главного лепестка, эллиптичность генерируемого излучения становится неодинаковой для различных направлений наблюдения $\theta, \varphi$.

При увеличении радиуса нелинейного слоя (например, на рис. $3, f$, где значение для радиуса частицы $k_{\omega} a=2.79$ взято из экспериментальной работы [6]) на диаграмме направленности появляются два главных лепестка большого размера и множество побочных, что объясняется свойствами предельных форм векторов $\mathbf{f}^{(\alpha \beta)}$ для больших значений $q^{(\alpha \beta)} a$ (часть I). При этом $A_{\mathrm{FB}}$ монотонно увеличивается и достигает практически единицы, начиная со значений $k_{\omega} a \approx 2.5$ (рис. $7, a$ ), что означает высокую направленность генерируемого излучения в переднюю полусферу. График $A_{\mathrm{RL}}\left(k_{\omega} a\right)$ также монотонно возрастает, пересекая ноль при $k_{\omega} a \approx 5.4$, и достигает примерно постоянных значений $A_{\mathrm{RL}} \approx 0.07$ при $k_{\omega} a>7$ (рис. 7, $b$ ). График асимметрии $A_{\mathrm{UD}}$ при увеличении $k_{\omega} a$ совершает колебания с постепенно уменьшающейся амплитудой (рис. 7,c). Возникающие колебания на графиках асимметрий можно объяснить появлением дополнительных несимметрично расположенных побочных лепестков на диаграммах направленности.

2) В случае анизотропии типа $\chi_{2}^{(2)}$ (рис. 2, $b$ ) интенсивность генерируемого излучения максимальна в направлениях $\theta \approx \pi / 2$ и убывает практически до нуля при $\theta=0$ или $\pi$. Увеличение угла раскрытия в диапазоне $0 \leq \gamma \leq 3$ почти не изменяет форму диаграммы направленности, дальнейшее возрастание $\gamma$ приводит к появлению двух симметричных лепестков в направлении передней и задней полусфер. Асимметрии FB- и RLдиаграмм направленности близки к нулю и слабо зависят от параметров $\arg \eta$ (рис. $5, a, 6, a)$ и $\varphi_{\text {in }}^{(2)}$ (рис. $\left.5, b, 6, b\right)$. Аналогичная закономерность проявляется на графике зависимости $A_{\mathrm{RL}}$ от $k_{\omega} a$ (рис. $\left.7, b\right)$. Асимметрия $A_{\mathrm{UD}}$ тождественно равна нулю при любых значениях параметров $\arg \eta, \varphi_{\text {in }}^{(2)}$ и $k_{\omega} a$ на графиках (рис. 5,c-7,c). На фоне остальных зависимостей для типа анизотропии $\chi_{2}^{(2)}$ выделяется зависимость $A_{\mathrm{FB}}$ от $k_{\omega} a$ (рис. 7,a), где мощность, излучаемая в переднюю полусферу, возрас- 

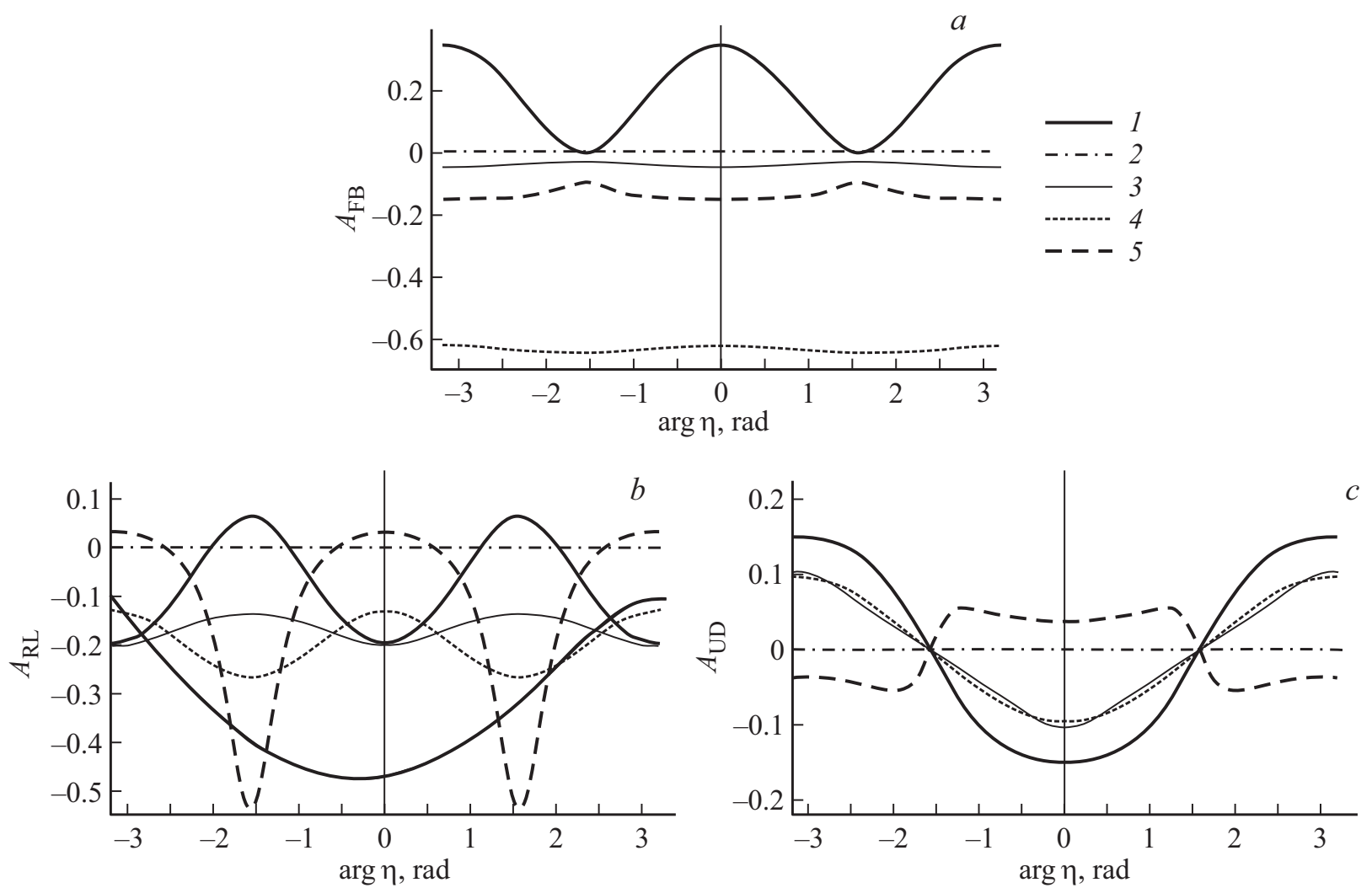

Рис. 5. Зависимость асимметрий генерируемого излучения от разности фаз падающих волн: $(a)$ вперед-назад, $(b)$ вправо-влево, (c) вверх-вниз. Типы анизотропии: $(1) \chi_{1}^{(2)},(2) \chi_{2}^{(2)},(3) \chi_{3}^{(2)},(4) \chi_{4}^{(2)},(5)$ малахитовый зеленый.

тает с небольшими осцилляциями и близка к 1 для больших размеров частицы. Изменение параметров $\eta$ $(|\eta|, \arg \eta), \varphi_{\text {in }}^{(1)}, \varphi_{\text {in }}^{(2)}, \xi, \sigma_{1}, \sigma_{2}$ на форму диаграммы направленности практически не влияет, но может приводить к ее повороту.

3) Для анизотропии $\chi_{3}^{(2)}$ (рис. 2,c) при варьировании параметров $\gamma, k_{\omega} a, \arg \eta, \varphi_{\text {in }}^{(1)}, \varphi_{\text {in }}^{(2)}, \sigma_{1}, \sigma_{2}$ характер изменения формы диаграммы направленности напоминает характер изменения для типа анизотропи $\chi_{1}^{(2)}$, что также проявляется подобием графиков для асимметрий $A_{\mathrm{RL}}$ (рис. $4, b-7, b)$ и $A_{\mathrm{UD}}$ (рис. $4, c-7, c$ ) и соответствующих графиков для типа анизотропии $\chi_{1}^{(2)}$. При малых $k_{\omega} a$ это можно объяснить двумя одинаковыми членами векторных функций $\mathbf{f}^{(\alpha \beta)}$ для типов анизотропии $\chi_{1}^{(2)}$ и $\chi_{3}^{(2)}$ (формула (34) части I).

Некоторые графики для $A_{\mathrm{FB}}$ имеют свои особенности. Асимметрия FB на графике зависимости от $\gamma$ (рис. $4, a$ ) равна нулю в точке $\gamma=0$, при этом на данном графике имеется один минимум $\left(A_{\mathrm{FB}} \approx-0.28\right.$ при $\left.\gamma \approx 2.05\right)$. Асимметрия FB при изменении $\arg \eta$ (рис. 5, $a$ ) почти не изменяется и приблизительно равна минус 0.03 во всей области изменения разности фаз. Асимметрия FB на графике зависимости от $\varphi_{\text {in }}^{(2)}$ (рис. 6, $a$ ) так же, как и для $\chi_{1}^{(2)}$, в области положительных значений параметра $\varphi_{\mathrm{in}}^{(2)}$ практически не меняется и близка к минус 0.04 , что объясняется значительно более симметричной формой диаграмм направленности относительно плоскости $O x y$ по сравнению с диаграммами направленности для типа анизотропии $\chi_{1}^{(2)}$.

4) Для кирального слоя (анизотропия $\chi_{4}^{(2)}$ ) (рис. $2, d$ ) характерно генерируемое излучение преимущественно в заднюю полусферу $\left(A_{\mathrm{FB}}=-0.622\right)$ в виде двух лепестков с линейной поляризацией. Асимметрия FB генерируемого излучения близка к минус 0.6 при $\gamma<0.96$ (рис. $4, a)$. При дальнейшем увеличении угла раскрытия асимметрия $\mathrm{FB}$ возрастает и приближается к нулю. График $A_{\mathrm{RL}}(\gamma)$ (рис. $\left.4, b\right)$ имеет вид, аналогичный виду соответствующих графиков для анизотропий $\chi_{1}^{(2)}$ и $\chi_{3}^{(2)}$, но с экстремумом $A_{\mathrm{RL}} \approx-0.17$ при $\gamma \approx 0.97$. Зависимость $A_{\mathrm{UD}}(\gamma)$ (рис. $4, c$ ) имеет минимум $A_{\mathrm{UD}} \approx-0.12$ при $\gamma \approx 0.92$ и максимум $A_{\mathrm{UD}} \approx 0.04$ при $\gamma \approx 2.60$, а при $\gamma \approx 2.10$ в верхнюю и нижнюю полусферы генерируется почти одинаковое количество энергии.

Изменение разности фаз падающих волн (рис. 5) практически не влияет на асимметрию $\mathrm{FB}\left(A_{\mathrm{FB}} \approx-0.63\right)$, а значения для асимметрии RL находятся в пределах $[-0.27,-0.13]$ с максимумами при $\arg \eta=-\pi, 0, \pi$ и минимумами при $\arg \eta= \pm \pi / 2$. График $A_{\mathrm{UD}}(\arg \eta)$ практически совпадает с аналогичным для анизотропии $\chi_{3}^{(2)}$.

Поворот направления поляризации второй волны (рис. 6) практически не приводит к изменениям асим- 

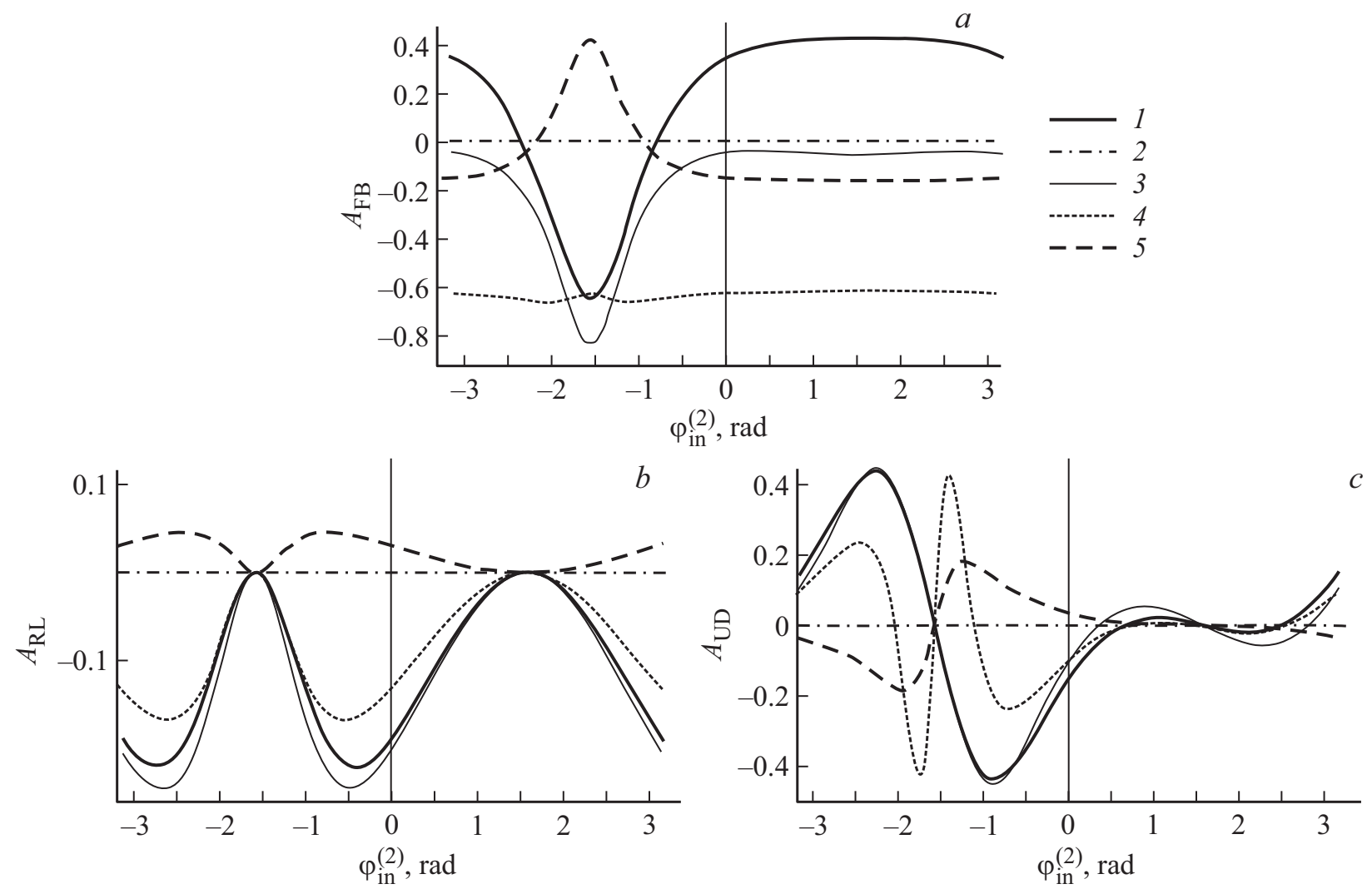

Рис. 6. Зависимость асимметрий генерируемого излучения от ориентации эллипса поляризации второй волны: (a) вперед-назад, (b) вправо-влево, (c) вверх-вниз. Типы анизотропии: $(1) \chi_{1}^{(2)},(2) \chi_{2}^{(2)},(3) \chi_{3}^{(2)},(4) \chi_{4}^{(2)},(5)$ малахитовый зеленый.

метрии $\mathrm{FB}\left(A_{\mathrm{FB}} \approx-0.62\right)$, но зависимости $A_{\mathrm{RL}}\left(\varphi_{\text {in }}^{(2)}\right)$ и $A_{\mathrm{UD}}\left(\varphi_{\mathrm{in}}^{(2)}\right)$ имеют по четыре экстремума, причем в области положительных $\varphi_{\text {in }}^{(2)}$ асиммметрия UD изменяется мало.

Графики для зависимостей асимметрий от радиуса слоя (рис. 7) имеют осциллирующий вид (для $A_{\mathrm{RL}}\left(k_{\omega} a\right)$ и $A_{\mathrm{UD}}\left(k_{\omega} a\right)$ наблюдаются заметные возмущения вблизи глобального максимума), но с ростом параметра $k_{\omega} a$ асимметрии стремятся к постоянным значениям: $A_{\mathrm{FB}} \rightarrow 0.64, A_{\mathrm{RL}} \rightarrow-0.03$.

5) Исследуем аналогичные зависимости асимметрий для случая малахитового зеленого, для которого экспериментально измеренные значения независимых компонент тензора нелинейной диэлектрической восприимчивости второго порядка таковы: $\chi_{1}^{(2)} / \chi_{2}^{(2)}=-0.806$, $\chi_{3}^{(2)} / \chi_{2}^{(2)}=-0.027, \chi_{4}^{(2)}=0$.

В этом случае в форме диаграммы направленности (рис. 2,e) просматриваются детали, характерные для рис. $2, a$ и $2, b$, соответствующие типам $\chi_{1}^{(2)}$ и $\chi_{2}^{(2)}$. Это можно объяснить тем, что значения коэффициентов $\chi_{1,2}^{(2)}$ значительно больше значений $\chi_{3,4}^{(2)}$.

Отметим некоторые особенности графиков асимметрий для малахитового зеленого. График зависимости $A_{\mathrm{FB}}$ от $\gamma$ (рис. 4, $a$ ) пересекает ось абсцисс в точке $\gamma \approx 2.17$ и $A_{\mathrm{FB}}$ максимальна при $\gamma \approx 2.81\left(A_{\mathrm{FB}} \approx 0.09\right)$.
Значения функции $A_{\mathrm{FB}}(\arg \eta)$ (ее график представлен на рис. $5, a)$ приблизительно равны минус 0.14 во всей области изменения параметра. Асимметрия RL при варьировании $\arg \eta$ (рис. $5, b)$ изменяется в очень больших пределах $\left(-0.53 \leq A_{\mathrm{RL}} \leq 0.03\right)$. Зависимость $A_{\mathrm{UD}}(\arg \eta)$ имеет два максимума при $\arg \eta \approx \pm 1.19$ и два минимума при $\arg \eta \approx \pm 1.96$. График $A_{\mathrm{FB}}\left(k_{\omega} a\right)$ (рис. $\left.7, a\right)$ близок к аналогичному графику для типа анизотропии $\chi_{2}^{(2)}$.

Возрастание угла раскрытия (рис. $8, a$ ) ведет к уменьшению лепестка, направленного в нижнюю полусферу. Варьирование параметра $|\eta|$ (рис. $8, b)$ в данном случае приводит к незначительному изменению формы диаграммы направленности и ее повороту. Изменение разности фаз волн первой гармоники до $\pi / 2$ приводит к преимущественной генерации в левую полусферу (рис. 8,c). На рис. $8, d$ изображена диаграмма направленности с наибольшей асимметрией в переднюю полусферу при варьировании параметра $\varphi_{\text {in }}^{(2)}$ и остальных параметрах, соответствующих (8). Если одна из падающих волн эллиптически (циркулярно) поляризована (рис. 8,e), то генерируется излучение с поляризацей, отличной от линейной. При увеличении радиуса частицы (рис. $8, f$ ) на диаграмме направленности появляются два главных лепестка и множество побочных, как и для анизотропии $\chi_{1}^{(2)}$, но они имеют несколько иную ориентацию (по отношению к углу наблюдения $\varphi$ ). 

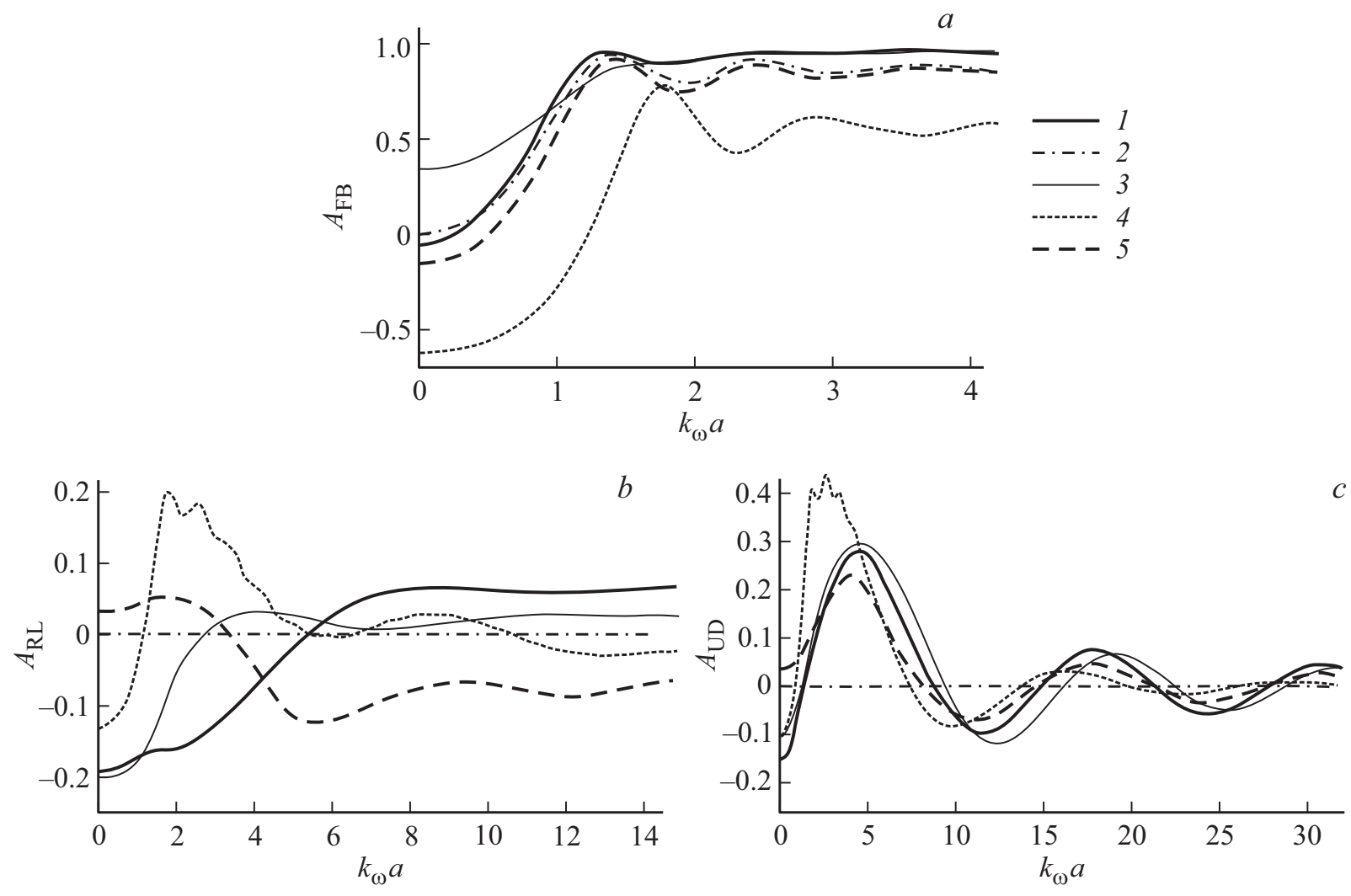

Рис. 7. Зависимость асимметрий генерируемого излучения от радиуса сферического слоя: $(a)$ вперед-назад, $(b)$ вправо-влево, (c) вверх-вниз. Типы анизотропии: $(1) \chi_{1}^{(2)},(2) \chi_{2}^{(2)},(3) \chi_{3}^{(2)},(4) \chi_{4}^{(2)},(5)$ малахитовый зеленый.

Рассмотрим особенности графиков для асимметрий (рис. 4-7).

1. На всех графиках зависимостей $A(\gamma)$ (рис. 4) значения асимметрий обращаются в ноль при встречном падении исходных волн $(\gamma=\pi)$. В случае сонаправленных волновых векторов источников $(\gamma=0)$ асимметрии $\mathrm{RL}$ и UD также равны нулю для всех типов анизотропии и для малахитового зеленого.

2. Зависимости асимметрий от разности фаз (рис. 5) имеют симметричный вид относительно замены $\arg \eta \rightarrow-\arg \eta$. Для асимметрий $\mathrm{FB}$ (рис. 5, $a$ ) и $\mathrm{RL}$ (рис. $5, b)$ одинаковые экстремумы наблюдаются при значениях $\arg \eta=-\pi, 0, \pi \quad$ и $\quad \arg \eta= \pm \pi / 2$. Значения функций на графиках зависимостей $A_{\mathrm{FB}}(\arg \eta)$ практически постоянны в случаях анизотропий $\chi_{2,3,4}^{(2)}$ и малахитового зеленого (рис. $5, a$ ). Для $A_{\mathrm{UD}}$ (рис. 5,c) и типов анизотропии $\chi_{1,3,4}^{(2)}$ минимум находится в точке $\arg \eta=0$, максимумы - в точке $\arg \eta= \pm \pi$. Также все асимметрии UD на описанных графиках обращаются в ноль при $\arg \eta= \pm \pi / 2$.

3. Значения асимметрии $\mathrm{FB}$ на графиках зависимости от $\varphi_{\text {in }}^{(2)}$ (рис. 6, $a$ ) почти не изменяются во всей области изменения параметра для типов анизотропии $\chi_{2}^{(2)}$ и $\chi_{4}^{(2)}$. При других значениях независимых компонент тензора $\chi_{i j k}^{(2)}$, рассмотренных в работе, асимметрии FB практически неизменны в области положительных $\varphi_{\text {in }}^{(2)}$ $\left(0 \leq \varphi_{\mathrm{in}}^{(2)} \leq \pi\right)$, а их графики имеют ярко выраженный экстремум при $\varphi_{\mathrm{in}}^{(2)}=-\pi / 2$. Зависимости $A_{\mathrm{RL}}\left(\varphi_{\mathrm{in}}^{(2)}\right)$ (рис. $6, b$ ) имеют по два минимума и максимума, обращаясь в ноль при $\varphi_{\mathrm{in}}^{(2)}= \pm \pi / 2$. Асимметрии UD (рис. 6,c) обращаются в ноль в этих же точках. Это объясняется симметрией задачи, а именно расположением волновых векторов падающих волн и их векторов поляризации. В этом случае пространственное распределение плотности мощности генерируемого излучения (диаграмма направленности) обладает осью симметрии, совпадающей с осью $O z$.

4. Зависимости асимметрий от радиуса нелинейного слоя (рис. 7) имеют осциллирующий вид, но с ростом параметра $k_{\omega} a$ стремятся к постоянным значениям. Для типов анизотропии $\chi_{1-3}^{(2)}$ и малахитового зеленого $A_{\mathrm{FB}} \rightarrow 1$ уже при $k_{\omega} a \approx 3$ (рис. 7, $a$ ), для $\chi_{4}^{(2)}$ получаем $A_{\mathrm{FB}} \rightarrow 0.64$, начиная с $k_{\omega} a \approx 4$. Графики зависимостей $A_{\mathrm{RL}}\left(k_{\omega} a\right)$ для каждого типа анизотропии стремятся к различным значениям, что наблюдается уже при $k_{\omega} a \approx 12$. На графиках зависимости асимметрий UD от $k_{\omega} a$ (рис. $\left.7, c\right)$ колебания вблизи нуля наблюдаются даже при $k_{\omega} a>50$, в чем можно убедиться, расширяя область построения графика в $2-3$ раза.

Нами было рассмотрено более 100 различных комбинаций значений параметров, перечисленных в (8), 


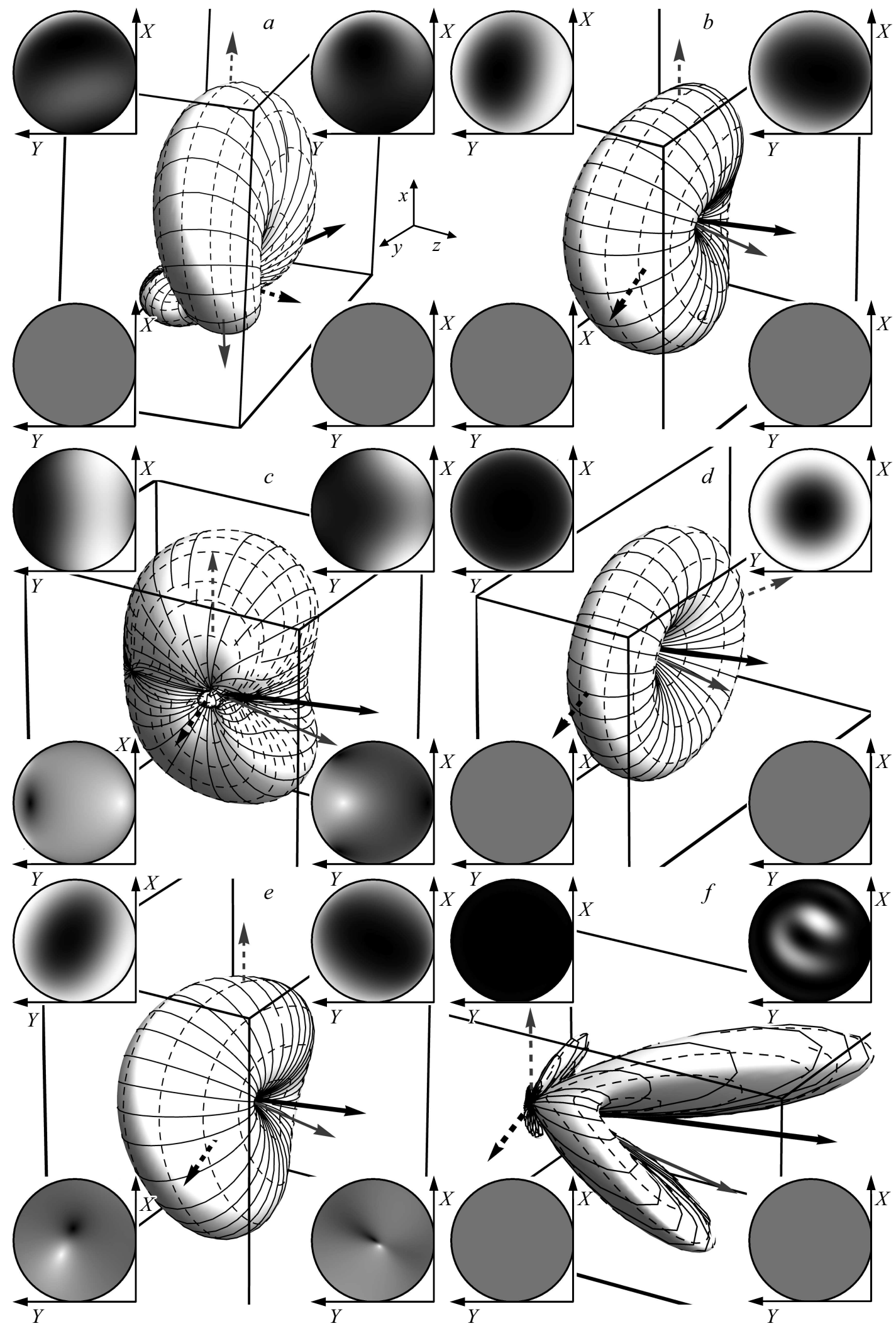

Рис. 8. Трехмерные нормированные диаграммы направленности для генерации второй гармоники-суммарной частоты от сферического слоя малахитового зеленого. Обозначения такие же, как и на рис. 2. Общие для всех диаграмм направленности параметры: $\sigma_{1}=0, \varphi_{\mathrm{in}}^{(1)}=\pi / 2$. Индивидуальные параметры построения: $(a) k_{\omega} a=0.1, \sigma_{2}=0, \eta=1, \varphi_{\mathrm{in}}^{(2)}=0, \gamma=2.5,(b) k_{\omega} a=0.1, \sigma_{2}=0$, $\eta=0.25, \varphi_{\text {in }}^{(2)}=0, \gamma=0.5,(c) k_{\omega} a=0.1, \sigma_{2}=0,|\eta|=1, \arg \eta=\pi / 2, \varphi_{\text {in }}^{(2)}=0, \gamma=0.5,(d) k_{\omega} a=0.1, \sigma_{2}=0, \eta=1, \varphi_{\text {in }}^{(2)}=-\pi / 2$, $\gamma=0.5,(e) k_{\omega} a=0.1, \sigma_{2}=1, \eta=1, \varphi_{\text {in }}^{(2)}=0, \gamma=0.5,(f) k_{\omega} a=2.79, \sigma_{2}=0, \eta=1, \varphi_{\text {in }}^{(2)}=0, \gamma=0.5$. 
при этом замечено, что для каждого из случаев 1)-5) характерна индивидуальная форма диаграммы направленности. При этом мы привели наиболее характерные диаграммы для малых размеров частиц.

\section{Эллиптичность генерируемого излучения}

В общем случае эллиптичность генерируемого излучения зависит от всех параметров задачи. Она также изменяется при варьировании направления наблюдения $\theta, \varphi$. Однако существуют сочетания параметров, при которых генерируется исключительно линейно поляризованное излучение во всех направлениях.

Используя явный вид векторов $\mathbf{f}^{(\alpha \beta)}$ (формула (20) части I), $\mathbf{e}^{(\alpha)}(2), \boldsymbol{v}^{(\alpha \beta)}(7)$, можно показать, что в случае падения двух линейно поляризованных электромагнитных волн, имеющих произвольные амплитуды и совпадающие или противоположные фазы в начале координат $(\operatorname{Im}[\eta]=0)$, вектор напряженности поля второй гармоники-суммарной частоты является чисто мнимым для некирального сферического слоя $\left(\forall \chi_{1-3}^{(2)}, \chi_{4}^{(2)}=0-\right.$ действительные числа) и действительным для кирального слоя $\left(\chi_{1-3}^{(2)}=0, \forall \chi_{4}^{(2)}\right.$ - действительное число). Как следствие, в каждом из этих случаев (некиральный слой, киральный слой) генерируемое излучение имеет линейную поляризацию во всех возможных направлениях наблюдения. Линейно поляризованное генерируемое излучение во всех направлениях при выполнении данных условий можно видеть на рис. $2, a$-e, рис. $3, a$, рис. $3, b$, рис. $3, d$, рис. $3, f$ рис. $8, a$, рис. $8, b$, рис. $8, d$, рис. $8, f$.

В частном случае сонаправленных волновых векторов в линейной поляризации генерируемого излучения легко убедиться, анализируя формулу (30) части I для эквивалентного вектора поляризации.

\section{Заключение}

В настоящей работе проведен графический анализ пространственного распределения генерируемого излучения второй гармоники-суммарной частоты в нелинейном сферическом слое путем построения диаграмм направленности и расчета асимметрий указанного распределения. Исследование проведено для четырех наиболее простых типов тензора диэлектрической восприимчивости второго порядка и тензора, соответствующего малахитовому зеленому [6].

Для каждого типа анизотропии характерны индивидуальные особенности изменения при варьировании параметров задачи. Однако повторяется следующая закономерность: увеличение радиуса нелинейного слоя приводит к поочередному появлению новых лепестков в нижней и верхней полусферах, что проявляется в виде осцилляций на графиках асимметрий, а также к росту направленности генерируемого излучения, на что указывает стремление большинства рассмотренных зависимостей $A_{\mathrm{FB}}\left(k_{\omega} a\right)$ к уровню $A_{\mathrm{FB}}=1$. При этом главные лепестки диаграмм направленности расположены в передней полусфере при больших значениях радиуса слоя.

Существование значений параметров, при которых асимметрии обращаются в ноль одновременно для всех типов тензора $\chi_{i j k}^{(2)}$, объясняется возникающей в этих случаях симметрией задачи. В остальных случаях обращение асимметрий в ноль является особенностью каждого типа анизотропии.

Для генерации второй гармоники и генерации суммарной частоты нелинейными слоями на поверхностях сферических и цилиндрических частиц доказано, что при падении линейно поляризованной электромагнитной волны на нелинейный слой, обладающий исключительно киральными или исключительно некиральными свойствами, генерируется только линейно поляризованное излучение $[2,10,11]$. Для генерации второй гармоники-суммарной частоты следует наложить дополнительное условие: разность фаз между падающими волнами должна быть равна нулю или $\pi$.

Описанные закономерности изменения пространственного распределения генерируемого поля подтверждают необходимость учета не только угла между волновыми векторами падающих волн, но и отношения их амплитуд, разности фаз, эллиптичности и ориентации эллипсов поляризации. Впоследствии полученные результаты могут быть использованы при планировании экспериментальных исследований нелинейной генерации в поверхностных слоях диэлектрических частиц, при изучении свойств адсорбированных на их поверхности молекул, а также при разработке методов управления эллиптичностью и распределением генерируемого излучения в пространстве.

\section{Финансирование работы}

Работа выполнена при финансовой поддержке Белорусского республиканского фонда фундаментальных исследований (грант Ф20М-011).

\section{Конфликт интересов}

Авторы заявляют, что у них нет конфликта интересов.

\section{Список литературы}

[1] de Beer A.G.F., Roke S., Dadap J.I. // JOSA. B. 2011. V. 28. N 6. P. 1374. doi 10.1103/PhysRevB.79.155420

[2] Шамына А.А., Капшай В.Н. // Опт. и спектр. 2018. T. 125. № 1. C. 71. doi 10.21883/OS.2018.07.46269.56-17; Shamyna A.A., Kapshai V.N. // Opt. Spectrosc. 2018. V. 125. N 1. P. 74. doi 10.1134/S0030400X1807024X

[3] Martorell J., Vilaseca R., Corbalan R. // Phys. Rev. A. 1997. V. 55. N 6. P. 4520. doi 10.1103/PhysRevA.55

[4] Viarbitskaya S., Kapshai V., van der Meulen P., Hansson T. // Phys. Rev. A. 2010. V. 81. N 5. P. 053850.

doi 10.1103/PhysRevA.81.053850 
[5] de Beer A.G.F., Roke S. // Phys. Rev. B. 2009. V. 79. N 15. P. 155420. doi 10.1103/PhysRevB.79.155420

[6] Wunderlich S., Schuerer B., Sauerbeck C., Peukert W., Peschel U. // Phys. Rev. B. 2011. V. 84. N 23. P. 235403. doi 10.1103/PhysRevB.84.235403

[7] Jen S.-H., Dai H.-L., Gonella G. // J. Phys. Chem. C. 2010. V. 114. N 10. P. 4302. doi 10.1021/jp910144c

[8] Daimon M., Masumura A. // Appl. Opt. 2007. V. 46. N 18. P. 3811. doi 10.1364/AO.46.003811

[9] Cao J., Heng Z., Wu L., Yang J.M. // Phys. Rev. D. 2010. V. 81. N 1. P. 014016. doi 10.1103/PhysRevD.81.014016.

[10] Капшай В.Н., Шамына А.А. // Опт. и спектр. 2017. T. 123. № 3. C. 416. doi 10.7868/S003040341709015X; Kapshai V.N., Shamyna A.A. // Opt. Spectrosc. 2017. V. 123. N 3. P. 440. doi 10.1134/S0030400X17090144

[11] Капшай В.Н., Шамына А.А. // Опт. и спектр. 2019. T. 126. № 6. C. 732. doi 10.21883/OS.2019.06.47766.375-18; Kapshai V.N., Shamyna A.A. // Opt. Spectrosc. 2019. V. 126. N 6. P. 653. doi 10.1134/S0030400X19060134 